\title{
FORMACIÓN EN EMPRENDIMIENTO PARA PERIODISTAS
}

\section{Pedro Aceituno-Aceituno, Carlos Bousoño-Calzón, José-Joaquín Escudero- Garzás y Francisco-José Herrera-Gálvez}

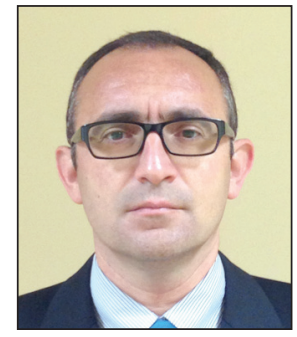

Pedro Aceituno-Aceituno es profesor contratado-doctor de creación y gestión de empresas de la Universidad a Distancia de Madrid (Udima) desde 2009 y coordinador-autor del manual de la asignatura. En la actualidad es investigador principal del proyecto IBGE de atracción y colaboración con los científicos españoles en el exterior financiado por el Ministerio de Empleo y Seguridad Social y del informe Innovacef de movilidad científica española. En 2003 se doctoró en ciencias económicas y empresariales por la Universidad Nacional de Educación a Distancia (UNED). http://orcid.org/0000-0001-9034-8673

Universidad a Distancia de Madrid Carretera de La Coruña, Km. 38,500. Vía de servicio, n. 15 28400 Collado Villalba, Madrid, España pedro.aceituno@udima.es

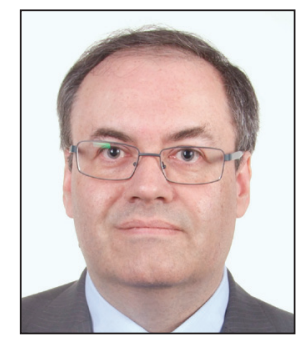

Carlos Bousoño-Calzón, profesor titular en la Universidad Carlos III de Madrid (UC3M), es responsable de la asignatura de innovación tecnológica, del grado de ingeniería en tecnologías de telecomunicación. Autor de decenas de trabajos en revistas y congresos internacionales y revisor de proyectos para entidades públicas. Entre 2007-2009 ha sido experto destacado en la Comisión Europea en la Unidad de Tecnologías Emergentes y Futuras (FET). Es doctor ingeniero de telecomunicación por la Universidad Politécnica de Madrid (1996). http://orcid.org/0000-0001-7065-5692

Universidad Carlos III de Madrid Av. de la Universidad, 30. 28911 Leganés (Madrid), España cbousono@tsc.uc3m.es

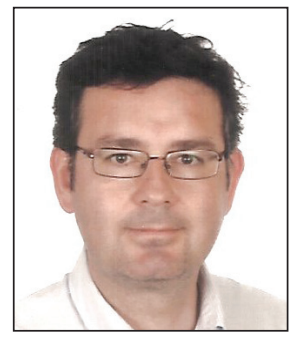

José-Joaquín Escudero-Garzás es profesor ayudante doctor en el Departamento de Teoría de la Señal y Comunicaciones (DTSC) de la Universidad Carlos III de Madrid (UC3M). Ha sido investigador en la Universidad Autónoma de Barcelona y en la Universidad de Virginia (EUA). Durante su trayectoria académica ha participado en proyectos nacionales e internacionales. Es doctor en multimedia y comunicaciones por la UC3M.

http://orcid.org/0000-0003-1456-7764

Universidad Carlos III de Madrid Av. de la Universidad, 30. 28911 Leganés (Madrid), España jescugar@tsc.uc3m.es

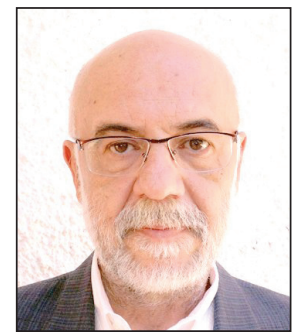

Francisco-José Herrera-Gálvez es ingeniero de telecomunicaciones por la Universidad Politécnica de Madrid (UPM) con más de 30 años de experiencia en el sector TIC. Durante este período ha ejercido varias responsabilidades, desde ingeniero de desarrollo a director de departamento en una multinacional del sector. Desde 2010 está centrado en la investigación del uso de los mercados de información como herramienta de agregación de información y sus aplicaciones en un entorno empresarial altamente dinámico como el actual.

http://orcid.org/0000-0002-0016-2697

Universidad Carlos III de Madrid Av. de la Universidad, 30. 28911 Leganés (Madrid), España fherrera54@gmail.com

\section{Resumen}

La superación de la crisis actual del sector periodístico y la urgencia de reformular los modelos de negocio hacia una mayor apertura a los usuarios y al entorno requiere la consecución de unos perfiles emprendedores en las futuras generaciones de periodistas. Para ello la formación resulta importante pero, de acuerdo con la literatura científica, sus efectos sobre el aumento de las intenciones emprendedoras no son del todo sólidos. Se presenta una experiencia de formación universitaria con el objetivo de proporcionar una mayor certeza de este incremento. Los resultados apoyan la validez de este tipo de formación en las intenciones emprendedoras y el alto nivel de incremento obtenido en los futuros periodistas. 


\title{
Palabras clave
}

Emprendimiento, Periodismo, Periodistas, Medios de comunicación, Modelos de negocio, Formación, Crisis, Encuestas.

\section{Title: Entrepreneurship training for journalists}

\begin{abstract}
Abstrac
Overcoming the newspaper industry crisis and addressing the urgent need to rethink business models to achieve a greater openness to users and the environment will require turning future generations of journalists into entrepreneurs. To meet this goal, training is important but, according to the scientific literature, its effects on increasing entrepreneurial intentions are not entirely convincing. This study presents a university programme with the aim to ensure this increased interest. The results support the validity of this type of training to generate entrepreneurial intentions and support a high level of increased entrepreneurship in future journalists.
\end{abstract}

\section{Keywords}

Entrepreneurship, Journalism, Journalists, Media, Business models, Training, Crisis, Surveys.

Aceituno-Aceituno, Pedro; Bousoño-Calzón, Carlos; Escudero-Garzás, José-Joaquín; Herrera-Gálvez, Francisco-José (2014). "Formación en emprendimiento para periodistas". El profesional de la información, julio-agosto, v. 23, n. 4, pp. 409-414.

http://dx.doi.org/10.3145/epi.2014.jul.09

\section{Introducción}

La situación de la prensa continúa siendo crítica. Problemas de viabilidad económica y despidos laborales en muchos países y excesivas incertidumbres sobre el futuro hicieron de 2009 el peor año de la historia de la prensa (Guallar, 2010). Estas circunstancias se mantienen en el presente con empresas periodísticas estranguladas por la crisis, disminución de sus ingresos y necesidad de afrontar este escenario tan desalentador (Guallar, 2013).

Los datos de 2012 de la industria global de la prensa, tanto de venta como de publicidad, muestran que los ingresos continúan cayendo en un porcentaje aproximado del $13 \%$ (127.172 millones de euros frente a los 145.803 de 2008). Las perspectivas son de mantenimiento en estos niveles como mínimo hasta 2017, ya que la continua expansión de los mercados de países emergentes (China, Brasil, India, Rusia, Oriente Próximo, Norte de África, México, Indonesia y Argentina) compensa el declive de mercados más maduros como los de Norteamérica o Europa Occidental y Central (PricewaterhouseCoopers, 2013).

En este contexto inestable y complejo, debido en gran parte a la aparición de internet como una nueva plataforma de medios de comunicación (Küng; Picard; Towse, 2008), resulta necesario y urgente refundar los modelos de negocio de la prensa para adaptarlos a la convergencia digital. Esta reformulación se enfrenta a obstáculos como la preeminencia de la cultura de la gratuidad en el acceso a noticias online o la intensificación de la competencia, que limitan el aumento de ingresos de las empresas periodísticas a pesar del incremento del consumo de información en internet (Casero-Ripollés, 2010). Sin embargo, gracias a las tecnologías digitales también se reducen las barreras de entrada al sector, por lo que la búsqueda de nuevos modelos de negocio abre un nuevo escenario de oportunidades para el emprendimiento entre los periodistas, que debe ser reforzado desde la formación universitaria (Casero-Ripollés; Cullell-March, 2013).
Desde las administraciones públicas y la universidad se ha ido prestando atención al emprendimiento, en paralelo a las transformaciones económicas acontecidas en el último tercio del siglo pasado (Ortega, 2012). Esto ha llevado al incremento de políticas públicas para su fomento mediante una educación que estimule el nivel de actividad económica (O’Connor, 2012), constituyéndose la formación en estos aspectos en una de las funciones básicas de la universidad (Yıldırım; Aşkun, 2012). Esta percepción positiva hacia la formación en emprendimiento es confirmada por Peterman y Kennedy (2003) y también por Wang y Wong (2004), aunque autores como Oosterbeek, Van Praag e ljsselstein (2010) y Souitaris, Zerbinati y Al-Laham (2007), no consideran que la educación en estas materias surta efecto sobre el incremento de las intenciones emprendedoras.

\section{Es necesario y urgente refundar los mo- delos de negocio de la prensa para adap- tarlos a la convergencia digital}

En este trabajo se hace referencia al concepto de emprendedor según la Real Academia Española de la Lengua (RAE), para la cual emprender consiste en "acometer y comenzar una obra, un negocio, un empeño, especialmente si encierran dificultad o peligro". Esto extiende el concepto de emprendedor no sólo a las personas que son capaces de crear una empresa, sino también a aquellas que comienzan nuevos proyectos o generan ideas en cualquier ámbito de una organización periodística.

La figura de emprendedor innovador (March-Chorda, 2010) está de acuerdo con lo que expone González-Esteban (2010), para quien los medios que sobrevivirán o triunfarán ante los nuevos escenarios mediáticos serán aquellos que reaccionen rápido, vayan donde está la gente, aprendan a enlazar, escuchar, cambiar, colaborar en la generación de 
contenidos con los usuarios y a compartir sus ingresos con ellos y, por último, comprendan la importancia de los blogs, la viralidad y la lógica de Google, que por la importancia del servicio que ofrece es capaz de unir a miles de consumidores, anunciantes y editores.

Esta idea está en consonancia con la utilización de modelos de negocio abiertos, que se pueden usar para la creación y captación de valor mediante la colaboración sistemática con socios externos, ya sea aprovechando las ideas procedentes desde el exterior de la empresa o proporcionando ideas o activos a terceros que no estén siendo utilizados por la empresa (Osterwalder; Pigneur, 2011). Sobre esto último, Filloux (2008) propone que la prensa proporcione servicios a las empresas (por ejemplo de asesoramiento de periodistas expertos en economía o de comunicación corporativa), lo que podría servir para ampliar los tradicionales ingresos de venta a los lectores y a los anunciantes de la prensa escrita (Sonnac, 2009) o de publicidad y de pago por contenidos de los cibermedios (Cea-Esteruelas, 2013).

Por todo lo expuesto es preciso que las personas que se van a dedicar a tareas periodísticas tengan un perfil emprendedor tanto si van a trabajar por cuenta propia como ajena. Es interesante aportar un caso cuyo objetivo sea confirmar el efecto positivo de la formación sobre el incremento de las intenciones emprendedoras de los futuros periodistas, lo que puede contribuir a enriquecer el conocimiento empírico sobre la generación y gestión de los medios de comunicación y de la empresa periodística como ciencia joven y multidisciplinar (Campos-Freire, 2010).

Se debe fomentar la figura del intraemprendedor o persona que desarrolla desde dentro de la compañía el emprendimiento

\section{Formación para el fomento del emprendimiento y metodología para su estudio}

Resulta interesante que este estudio se realice en una universidad española, debido a que las condiciones en que los futuros periodistas tendrán que realizar su trabajo no son las más adecuadas, como se pone de manifiesto en el Informe anual de la profesión periodística 2012 (Palacios-Llanos, 2012). Los periodistas consultados en dicho informe afirman que el principal problema de la profesión es el aumento del paro y la precariedad laboral $(51,4 \%)$, seguido de las malas retribuciones (12,3\%), por lo que el autoempleo o la generación de nuevos modelos de negocio dentro de las empresas existentes se muestran como alternativas válidas para ejercer esta profesión en España. Este último aspecto también es muy importante y necesario, pues las empresas españolas en general carecen de pautas directivas que estimulen un adecuado flujo de conocimiento desde los empleados a la dirección (Coduras-Martínez, 2012), por lo que la figura del intraemprendedor o persona que desarrolla desde dentro el emprendimiento en la compañía debe ser fomentada y reconocida en mayor medida.
El caso de estudio se ha realizado sobre las asignaturas "Creación y gestión de empresas" e "Introducción a la organización de empresas" de la Universidad a Distancia de Madrid (Udima) en los cursos 2012-2013 y 2013-2014. En relación con la primera asignatura, algunos autores como Fernández-Laviada, Rueda-Sampedro y Herrero-Crespo (2011) aconsejan que estos cursos presenten situaciones ajustadas a la realidad empresarial. Para ello los alumnos han realizado proyectos en colaboración con empresarios y emprendedores con los que interactúan dentro de los foros virtuales del aula o planes de negocios con una clara vocación social de generar puestos de trabajo.

Un porcentaje elevado (85\%) de los expertos consultados en el Libro blanco de la iniciativa emprendedora (Alemany et al., 2011) opina que desde la universidad no se proporciona una adecuada formación para estimular las intenciones de emprendimiento. Por ello es necesario que en materias como "Introducción a la organización de empresas" se impulsen estas intenciones. En este sentido, los alumnos de esta materia y de "Creación y gestión de empresas" han realizado proyectos en colaboración con empresarios y emprendedores.

Además los estudiantes de ambas asignaturas han participado en una red social interna para exponer noticias de emprendimiento, en sesiones de videoconferencia relativas al tema, han visionado vídeos de experiencias de empresarios y emprendedores, y han participado en "mercados de información". Éstos pueden ser definidos como un dispositivo de inteligencia colectiva como los que se ha venido empleando en el ámbito laboral de grandes empresas como Google o Microsoft, para estimular la creatividad y compromiso de los empleados (Thompson, 2012). En la experiencia que se está exponiendo, dichos mercados han actuado con una bolsa de valores ficticia en la que los alumnos podían comprar y vender las acciones de los proyectos de los empresarios y de los emprendedores, lo que puede ayudar a éstos en la evaluación y mejora de sus proyectos, permitiendo a su vez que los estudiantes afronten una actividad adicional, que resulta innovadora y de colaboración con la realidad empresarial.

Esta formación favorece que el estudiante pueda aproximarse a la realidad, lo que ayuda a que el alumno pueda aplicar sus conocimientos a la vez que está estudiando. También le permite profundizar en el funcionamiento de modelos de negocio abiertos como los citados anteriormente (Osterwalder; Pigneur, 2011), ya que con esta educación se favorece la interactuación, la generación y desarrollo de las ideas y la colaboración entre todos los participantes en el aula. Por la importancia de estos aspectos para el desarrollo futuro de cualquier empresa, se ha fomentado su uso no sólo entre los estudiantes del Grado de Periodismo, sino también entre los de otras titulaciones: Administración y dirección de empresas, Ciencias del trabajo y recursos humanos, Derecho, Empresas y actividades turísticas, Ingeniería informática, Magisterio en educación primaria y Psicología.

Para comprobar la variación de las intenciones emprendedoras debido al efecto de la formación, se han utilizado dos encuestas (una antes de recibir la formación y otra después), siguiendo el modelo de Shapero (1975), bajo el enfoque de Peterman y Kennedy (2003), que plantea una 
Tabla 1. Número y porcentaje de alumnos por grado

\begin{tabular}{|l|c|c|}
\hline \multicolumn{1}{|c|}{ Grado } & Número de alumnos & $\%$ \\
\hline $\begin{array}{l}\text { Administración y dirección de } \\
\text { empresas }\end{array}$ & 67 & 28,3 \\
\hline $\begin{array}{l}\text { Ciencias del trabajo y recursos } \\
\text { humanos }\end{array}$ & 41 & 17,3 \\
\hline Derecho & 75 & 31,6 \\
\hline $\begin{array}{l}\text { Empresas y actividades turís- } \\
\text { ticas }\end{array}$ & 10 & 4,2 \\
\hline Ingeniería informática & 9 & 3,8 \\
\hline $\begin{array}{l}\text { Magisterio en educación } \\
\text { primaria }\end{array}$ & 31 & 0,4 \\
\hline Periodismo & 3 & 13,1 \\
\hline Psicología & 237 & 1,3 \\
\hline Total & & 100 \\
\hline
\end{tabular}

cuestión análoga al objeto de este trabajo bajo el programa Young Achievement Australia (YAA) dedicado a la formación en emprendimiento para colectivos más jóvenes.

Las intenciones de emprendimiento se componen de dos variables: la deseabilidad y la factibilidad percibidas para emprender un negocio

Según este modelo las intenciones de emprendimiento se componen de dos variables: la deseabilidad y la factibilidad percibidas para emprender un negocio. Como se observa en las tablas 2 y 3 , para definir la primera variable se utilizan tres preguntas sobre la obtención de resultados personalmente deseables de la acción de emprender, y para la segunda 5 preguntas para establecer el grado de posibilidades de éxito que considera el emprendedor. Las preguntas son valoradas con una escala de Likert de siete puntos cada una, en la que los valores más altos expresan una deseabilidad y una factibilidad peores, y los valores bajos, mejores.

\section{Resultados}

La muestra objeto de estudio se compone de 237 estudiantes, que han recibido formación en emprendimiento durante dos cursos académicos (2012-2013 y 2013-2014). En la tabla 1 se expone el número de alumnos de cada grado, que determina aquí los perfiles de estudiantes.

Para conocer si se ha producido un efecto positivo de la formación sobre el incremento de las intenciones emprendedoras y establecer el nivel comparado de estas intenciones en los futuros periodistas con respecto a los estudiantes del resto de grados, la metodología anteriormente expuesta se complementa con el estudio para diferentes grados similar al de Arias-Aranda, Haro-Domínguez y Romerosa-Martínez (2010), en el que se analiza la varianza (anova), cuyos resultados para el grado de periodismo y para la agregación del resto de grados se exponen en las tablas 2 y 3.

En la primera encuesta (tabla 2), antes de impartirse la formación, los dos colectivos tienen una alta deseabilidad (recuérdese que valores más bajos suponen mejores intenciones emprendedoras). El anova no muestra aquí diferencias significativas entre los dos colectivos. Sin embargo la factibilidad percibida por los periodistas es peor que el resto de los grados, con diferencias estadísticamente significativas en cuatro de las cinco preguntas. Estos resultados sugieren que lo que fundamentalmente precisan estos alumnos es percibir que su proyecto es realmente viable para emprenderlo, dado lo cual los aspectos más relacionados con la factibilidad son aquellos en los que la formación debe ser más efectiva.

En la segunda encuesta (tabla 3), tras la formación se observa que los dos colectivos mejoran sus percepciones tanto en

Tabla 2. Cuestionario y escalas utilizadas en la primera encuesta

\begin{tabular}{|c|c|c|c|c|c|c|}
\hline Pregunta & \multicolumn{2}{|c|}{ Periodismo } & \multicolumn{2}{|c|}{ Resto de grados } & \multirow{2}{*}{$\begin{array}{l}\text { Anova } \\
\text { F }\end{array}$} & \multirow{2}{*}{$\begin{array}{c}\text { Anova } \\
\text { probabili- } \\
\text { dad }\end{array}$} \\
\hline Factibilidad percibida & Media & $\begin{array}{l}\text { Desviación } \\
\text { típica }\end{array}$ & Media & $\begin{array}{l}\text { Desviación } \\
\text { típica }\end{array}$ & & \\
\hline $\begin{array}{l}\text { 1. ¿Será fácil iniciarlo? } \\
\text { (Muy fácil = 1; Muy difícil = 7) }\end{array}$ & 4,29 & 1,55 & 4,09 & 1,46 & 0,47 & 0,50 \\
\hline $\begin{array}{l}\text { 2. ¿Estás seguro del éxito? } \\
\text { (Muy seguro del éxito = } 1 \text {; Muy seguro del fracaso = 7) }\end{array}$ & 4,03 & 1,70 & 3,45 & 1,32 & 4,81 & 0,03 \\
\hline $\begin{array}{l}\text { 3. ¿Crees que el trabajo sería excesivo? } \\
(\text { El trabajo no es excesivo }=1 ; \text { Muy excesivo el trabajo }=7 \text { ) }\end{array}$ & 5,48 & 1,34 & 4,85 & 1,44 & 5,21 & 0,02 \\
\hline $\begin{array}{l}\text { 4. ¿Sabes lo suficiente como para iniciar un negocio? } \\
\text { (Saber todo }=1 \text {; Saber nada }=7 \text { ) }\end{array}$ & 4,81 & 1,38 & 4,17 & 1,55 & 4,65 & 0,03 \\
\hline $\begin{array}{l}\text { 5. ¿Estás seguro de ti mismo? } \\
\text { (Muy seguro de mí mismo = } 1 \text {; Muy inseguro de mí mismo = 7) }\end{array}$ & 3,32 & 1,87 & 2,71 & 1,56 & 3,91 & 0,05 \\
\hline \multicolumn{7}{|l|}{ Deseabilidad percibida } \\
\hline $\begin{array}{l}\text { 6. ¿Te encantaría emprender un nuevo negocio? } \\
\text { (Me encantaría hacerlo }=1 \text {; Odiaría hacerlo }=7 \text { ) }\end{array}$ & 2,71 & 1,74 & 2,35 & 1,50 & 1,44 & 0,23 \\
\hline $\begin{array}{l}\text { 7. ¿Estarías muy preocupado por ello? } \\
\text { (Nada preocupado = } 1 \text {; Muy preocupado = 7) }\end{array}$ & 5,68 & 1,40 & 5,33 & 1,61 & 1,29 & 0,26 \\
\hline $\begin{array}{l}\text { 8. ¿Estas entusiasmado con la idea de iniciar un negocio? } \\
\text { (Muy entusiasmado }=1 \text {; Muy poco entusiasmado }=7 \text { ) }\end{array}$ & 2,81 & 1,97 & 2,82 & 1,70 & 0 & 0,98 \\
\hline
\end{tabular}


Tabla 3. Cuestionario y escalas utilizadas en la segunda encuesta

\begin{tabular}{|c|c|c|c|c|c|c|}
\hline Pregunta & \multicolumn{2}{|c|}{ Periodismo } & \multicolumn{2}{|c|}{ Resto de grados } & \multirow{2}{*}{$\begin{array}{l}\text { Anova } \\
\quad F\end{array}$} & \multirow{2}{*}{$\begin{array}{c}\text { Anova } \\
\text { probabili- } \\
\text { dad }\end{array}$} \\
\hline Factibilidad percibida & Media & $\begin{array}{l}\text { Desviación } \\
\text { típica }\end{array}$ & Media & $\begin{array}{l}\text { Desviación } \\
\text { típica }\end{array}$ & & \\
\hline $\begin{array}{l}\text { 1. ¿Será fácil iniciarlo? } \\
\text { (Muy fácil = } 1 \text {; Muy difícil = 7) }\end{array}$ & 2,62 & 1,37 & 3,92 & 1,39 & 21,42 & 0,00 \\
\hline $\begin{array}{l}\text { 2. ¿Estás seguro del éxito? } \\
\text { (Muy seguro del éxito = } 1 \text {; Muy seguro del fracaso = 7) }\end{array}$ & 2,41 & 1,12 & 3,36 & 1,21 & 15,33 & 0,00 \\
\hline $\begin{array}{l}\text { 3. ¿Crees que el trabajo sería excesivo? } \\
(\text { El trabajo no es excesivo }=1 ; \text { Muy excesivo el trabajo }=7 \text { ) }\end{array}$ & 4,10 & 2,08 & 4,82 & 1,53 & 4,82 & 0,03 \\
\hline $\begin{array}{l}\text { 4. ¿Sabes lo suficiente como para iniciar un negocio? } \\
\text { (Saber todo }=1 ; \text { Saber nada }=7 \text { ) }\end{array}$ & 2,79 & 1,21 & 3,54 & 1,26 & 8,85 & 0,00 \\
\hline $\begin{array}{l}\text { 5. ¿Estás seguro de ti mismo? } \\
\text { (Muy seguro de mí mismo }=1 ; \text { Muy inseguro de mí mismo = } 7 \text { ) }\end{array}$ & 2,00 & 1,16 & 2,44 & 1,32 & 2,89 & 0,09 \\
\hline \multicolumn{7}{|l|}{ Deseabilidad percibida } \\
\hline $\begin{array}{l}\text { 6. ¿Te encantaría emprender un nuevo negocio?. } \\
\text { (Me encantaría hacerlo }=1 \text {; Odiaría hacerlo = 7) }\end{array}$ & 1,76 & 1,09 & 2,34 & 1,52 & 3,85 & 0,05 \\
\hline $\begin{array}{l}\text { 7. ¿Estarías muy preocupado por ello? } \\
\text { (Nada preocupado }=1 \text {; Muy preocupado =7) }\end{array}$ & 4,03 & 1,92 & 4,95 & 1,66 & 7,12 & 0,01 \\
\hline $\begin{array}{l}\text { 8. ¿Estas entusiasmado con la idea de iniciar un negocio? } \\
\text { (Muy entusiasmado }=1 \text {; Muy poco entusiasmado }=7 \text { ) }\end{array}$ & 1,97 & 1,27 & 2,77 & 1,68 & 6,09 & 0,01 \\
\hline
\end{tabular}

deseabilidad como en factibilidad. Los cambios en la deseabilidad son más relevantes para los periodistas cuyas diferencias son ahora estadísticamente significativas en todas las respuestas. Resultan realmente destacables las mejoras que se han producido para este grado en todas las preguntas de factibilidad, con incrementos bastante superiores a un punto y en algunos casos, como el de la pregunta relacionada con el conocimiento para abrir un negocio, superiores a dos puntos.

\section{Conclusiones}

Los resultados muestran la efectividad de la formación, pues ha logrado incrementar para los dos colectivos las intenciones emprendedoras tanto en la deseabilidad como muy especialmente en la factibilidad, que era el aspecto en el que más se precisaba de su acción, con aumentos dentro de esta última muy destacables para ambos grupos en relación con la aportación de conocimientos para abrir un negocio.

Los resultados refrendan el efecto positivo del enfoque de Peterman y Kennedy (2003) y de la importancia que tiene una formación integral que atienda a las dos variables (deseabilidad y factibilidad).

En comparación con el resto de los grados, esta formación ha fortalecido en mayor medida las intenciones emprendedoras de los alumnos de periodismo en los dos aspectos, pero muy particularmente en la factibilidad. El incremento se apoya en dos elementos: 1) una mayor concienciación de la crisis del sector, que hace más necesario emprender si se pretende ejercer esa profesión, y 2) la presentación de la opción emprendedora estimulada por una formación integral que les ha proporcionado ajuste a la realidad, espíritu de colaboración, ánimo y asesoramiento personalizado, que son aspectos que benefician tanto al deseo de ser empresario (obtención de un mayor autonomía o crecimiento personal, por ejemplo) como a las de poseer una mayor certeza de obtener un éxito con el emprendimiento.

Aunque los resultados estadísticos sobre los que se apoyan estas conclusiones son robustos en relación con la efectividad de la formación en emprendimiento, el estudio presenta la limitación de haberse realizado sobre una pequeña muestra de estudiantes del grado de periodismo, por lo que en futuros estudios se debería ampliar esta muestra para ratificar la validez de los resultados.

Las conclusiones que se extraen del estudio corroboran la hipótesis de que la formación en emprendimiento tiene un efecto positivo sobre las intenciones emprendedoras de los estudiantes y muy especialmente de los de periodismo, lo que puede convertirse en un elemento esencial para que el sector supere su crisis y pueda seguir cumpliendo su importante función en beneficio de la sociedad.

\section{Agradecimientos}

A todos los estudiantes, empresarios y emprendedores que han participado en el estudio, y muy especialmente, a los periodistas emprendedores de la Udima. Asimismo, también se agradece el patrocinio de este estudio por Novanca. Por último, agradecemos a la Udima todos los medios económicos, materiales y humanos dispuestos para este trabajo a través de su Programa de Creación y Consolidación de Grupos de Investigación (referencia UI2011-2).

\section{Bibliografía}

Alemany, Luisa; Álvarez, Claudia; Planellas, Marcel; Urbano, David (2011). Libro blanco de la iniciativa emprendedora en España. Barcelona: Esade.

http://www.cise.es/wp-content/uploads/2013/03/9_ LBIEE_Documento-Final.pdf

Arias-Aranda, Daniel; Haro-Domínguez, Carmen; Romerosa-Martínez, María-Mercedes (2010). “Un enfoque innovador del proceso de enseñanza-aprendizaje en la dirección de empresas: el uso de simuladores en el ámbito universitario". Revista de educación, v. 353, pp. 707-721.

http://www.revistaeducacion.educacion.es/re353/re353_27.pdf 
Campos-Freire, Francisco (2010). "Los nuevos modelos de gestión de las empresas mediáticas". Estudios sobre el mensaje periodístico, v. 16, pp. 13-30.

http://revistas.ucm.es/index.php/ESMP/article/view/12163

Casero-Ripollés, Andreu (2010). "Prensa en internet: nuevos modelos de negocio en el escenario de la convergencia". El profesional de la información, v. 19, n. 6, pp. 595-601. http://repositori.uji.es/xmlui/bitstream/handle/10234/22432/46493. pdf?sequence $=1$

http://dx.doi.org/10.3145/epi.2010.nov05

Casero-Ripollés, Andreu; Cullell-March, Cristina (2013). "Periodismo emprendedor. Estrategias para incentivar el autoempleo periodístico como modelo de negocio". Estudios sobre el mensaje periodístico, v. 19, pp. 681-690.

http://revistas.ucm.es/index.php/ESMP/article/view/42151

Cea-Esteruelas, María-Nereida (2013). “Cybermedia economics: revenue model and sources of financing". El profesional de la información, v. 22, n. 4, pp. 353-361.

http://www.elprofesionaldelainformacion.com/ contenidos/2013/julio/12_esp.pdf

http://dx.doi.org/10.3145/epi.2013.jul.12

Coduras-Martínez, Alicia (2012). "La capacidad emprendedora en la población española: situación actual y retos a afrontar". Economistas, v. 132, pp. 53-65.

http://goo.gl/h51FnW

Fernández-Laviada, Ana; Rueda-Sampedro, María Inés; Herrero-Crespo, Ángel (2011). "Estudio de la actitud emprendedora de los estudiantes universitarios de la facultad de CC. EE. y empresariales de Cantabria". En: XVI Congreso de la Asociación Española de Contabilidad y Administración de Empresas (AECA), pp. 1-22.

http://www.aeca1.org/pub/on_line/comunicaciones_xvicongresoaeca/cd/36f.pdf

Filloux, Frédéric (2008). "The economics of moving from print to online: lose one hundred, get back eight". Monday note. Media, tech, business models, 20 Sept.

http://www.mondaynote.com/2008/09/29/the-economics-ofmoving-from-print-to-online-lose-one-hundred-get-back-eight

González-Esteban, José-Luis (2010). “Auge y caída de Soitu, un ejemplo de medio nativo digital en España". Comunicación y sociedad, v. XXIII, n. 2, pp. 267-288.

http://www.unav.es/fcom/comunicacionysociedad/es/ articulo.php?art_id=371

Guallar, Javier (2010). “Prensa digital en 2009". Anuario ThinkEPI, v. 4, pp. 165-173.

Guallar, Javier (2013). "Prensa digital en 2011-2012". Anuario ThinkEPI, v. 7, pp. 194-199.

Küng, Lucy; Picard, Robert G.; Towse, Ruth (2008). The internet and the mass media. Londres: Sage Publications. ISBN: 9781412947343

March-Chorda, Isidre (2010). "Las claves del éxito en nuevas compañías innovadoras según los propios emprendedores". Dirección y organización, v. 21, pp. 167-176.

http://revistadyo.es/index.php/dyo/article/viewFile/303/303
O'Connor, Allan (2012). "A conceptual framework for entrepreneurship education policy: meeting government and economic purposes". Journal of business venturing, July, v. 28, n. 4, pp. 546-563. http://dx.doi.org/10.1016/j.jbusvent.2012.07.003

Oosterbeek, Hessel; Van-Praag, Mirjam; ljsselstein, Auke (2010). "The impact of entrepreneurship education on entrepreneurship skills and motivation". European economic review, v. 54, pp. 442-454.

http://dx.doi.org/10.1016/j.euroecorev.2009.08.002

Ortega, Iñaki (2012). “Política pública de promoción de la capacidad emprendedora en España”. Economistas, v. 132, pp. 66-71. http://www.inakiortega.com/2012/09/las-politicas-publicasde-promocion-de.html

Osterwalder, Alexander; Pigneur, Ives (2011). Generación de modelos de negocio. Barcelona: Deusto Grupo Planeta. ISBN: 9788423427994

Palacios-Llanos, Luis (2012). Informe anual de la profesión periodística 2012. Asociación de la prensa de Madrid. http://www.apmadrid.es/images/stories/Informe\%20 APM\%202012.pdf

Peterman, Nicole; Kennedy, Jessica (2003). "Enterprise education: influencing students' perceptions of entrepreurship". Entrepreurship theory and practice, v. 28, n. 2, pp. 129-144. http://dx.doi.org/10.1046/j.1540-6520.2003.00035.x

PricewaterhouseCoopers (2013). Global entertainment and media Outlook: 2013-2017.

https://www.pwc.com/outlook

Real Academia Española (2013)

http://lema.rae.es/drae/?val=emprender

Shapero, Albert (1975). "The displaced, uncomfortable entrepreneur". Psychology today, v. 9, pp. 83-88.

Sonnac, Nathalie (2009). "L'économie de la presse: vers un nouveau modèle d'affaires". Les cahiers du journalisme, automne, n. 20, pp. 22-43.

http://www.cahiersdujournalisme.net/cdj/pdf/20/01_SONNAC.pdf

Souitaris, Vangelis; Zerbinati, Stefania; Al-Laham, Andreas (2007). "Do entrepreneurship programmes raise entrepreneurial intention of science and engineering students? The effect of learning, inspiration and resources". Journal of business venturing, v. 22, pp. 566-591.

http://dx.doi.org/10.1016/j.jbusvent.2006.05.002

Thompson, Donald (2012). Oracles: how prediction markets turn employees into visionaries. EUA: Harvard Business School Publishing. ISBN: 9781422183175

Wang, Clement K.; Wong, Poh-Kam (2004). "Entrepreneurial interest of university students in Singapore". Technovation, v. 24, pp. 163-172. http://dx.doi.org/10.1016/S0166-4972(02)00016-0

Yıldırım, Nihan; Aşkun, Olcay-Bige (2012). "Entrepreneurship intentions of public universities in Turkey: going beyond education and research?". Procedia-Social and behavioral sciences, v. 58, pp. 953-963.

http://dx.doi.org/10.1016/j.sbspro.2012.09.1074 